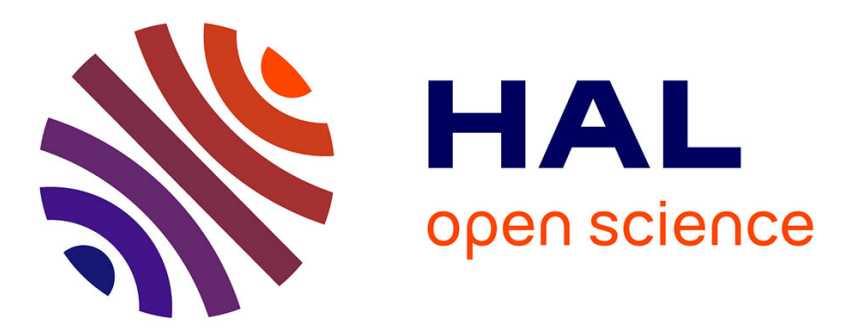

\title{
Interatomic Coulombic decay widths of helium trimer: A diatomics-in-molecules approach
}

\author{
Nicolas Sisourat, Sévan Kazandjian, Aurélie Randimbiarisolo, Přemysl \\ Kolorenč
}

\section{- To cite this version:}

Nicolas Sisourat, Sévan Kazandjian, Aurélie Randimbiarisolo, Přemysl Kolorenč. Interatomic Coulombic decay widths of helium trimer: A diatomics-in-molecules approach. Journal of Chemical Physics, 2016, 144 (8), pp.084111. 10.1063/1.4942483 . hal-01291989

\section{HAL Id: hal-01291989 \\ https://hal.sorbonne-universite.fr/hal-01291989}

Submitted on 22 Mar 2016

HAL is a multi-disciplinary open access archive for the deposit and dissemination of scientific research documents, whether they are published or not. The documents may come from teaching and research institutions in France or abroad, or from public or private research centers.
L'archive ouverte pluridisciplinaire HAL, est destinée au dépôt et à la diffusion de documents scientifiques de niveau recherche, publiés ou non, émanant des établissements d'enseignement et de recherche français ou étrangers, des laboratoires publics ou privés. 


\title{
Interatomic Coulombic Decay widths of helium trimer : a diatomics-in-molecules approach
}

\author{
Nicolas Sisourat, ${ }^{1}$ Sévan Kazandjian, ${ }^{1}$ Aurélie Randimbiarisolo, ${ }^{1, *}$ and Přemysl Kolorenč ${ }^{2}$ \\ ${ }^{1}$ Sorbonne Universités, UPMC Univ Paris 06, CNRS, \\ Laboratoire de Chimie Physique Matière et Rayonnement, F-75005, Paris, France \\ ${ }^{2}$ Charles University in Prague, Faculty of Mathematics and Physics, \\ Institute of Theoretical Physics, V Holešovičkách 2, 18000 Prague, Czech Republic
}

(Dated: March 21, 2016)

\begin{abstract}
We report a new method to compute the Interatomic Coulombic Decay (ICD) widths for large clusters which relies on the combination of the projection-operator formalism of scattering theory and the diatomics-in-molecules approach. The total and partial ICD widths of a cluster are computed from the energies and coupling matrix elements of the atomic and diatomic fragments of the system. The method is applied to the helium trimer and the results are compared to fully $a b$ initio widths. A good agreement between the two sets of data is shown. Limitations of the present method are also discussed.
\end{abstract}

\footnotetext{
* Nicolas.Sisourat@upmc.fr
} 


\section{INTRODUCTION}

Resonance phenomena appear in many physical, chemical and biological processes [1], for example in atom-molecule or electron-molecule collisions, photoionization and autoionization events. Even though resonance states have been investigated for decades [2], computing their properties is still a challenging task.

Among the resonance phenomena, the Interatomic Coulombic Decay (ICD) effect has attracted considerable attention in the last decade. ICD is an ultrafast non-radiative electronic decay process for excited atoms or molecules embedded in a chemical environment, like in a cluster. Via this process, the excited species can get rid of the excess energy, which is transferred to one of the neighbors and ionizes it. The ICD process was predicted in the late 90's by Cederbaum et al. [3]. It was experimentally demonstrated about 10 years ago on the example of neon clusters by Marburger et al. [4] and Jahnke et al. [5]. Since then, it was shown that ICD is a general process, taking place in a large variety of systems, like hydrogen bonded $[6,7]$ or van der Waals clusters [8-11]. It has been observed mainly after photoionization or photoexcitation but has also been experimentally demonstrated after electron [12] and ion impact [13-15]. ICD was first predicted and observed after ionization in the inner-valence shell, but it was also demonstrated (i) after two-electron processes like simultaneous ionization-excitation [16, 17] and double ionization or excitation [18, 19], (ii) in cascades after Auger [20-23] and resonant Auger [24, 25].

The lifetime of a resonant state, or turning from the time to the energy domain the width of this state is an important property to characterize the resonance. General quantum mechanical equations for computing the decay widths are known but are only applicable to small systems. The main difficulty stems from the fact that one has to account both for the many-body and for the scattering aspects of the decay phenomenon. Several computational approaches have been developed and used to calculate ICD widths [26-29]. Among them, methods based on Complex Absorbing Potential (CAP) provide only total widths. Partial widths can be obtained using Wigner-Weisskopf method. However, this approach is based on the lowest-order perturbation theory and thus gives only estimates for the widths. To date, only the Fano-ADC method [30-32] provides reliable partial ICD widths which are necessary for full theoretical description of the process. This ab initio method is, however, computationally expensive and not applicable to really large systems. 
We report here on a simpler approach based on the diatomics-in-molecules (DIM) technique [33] for the computations of the ICD widths in rare-gas clusters. In the standard DIM approach, the Hamiltonian matrix elements of a system are evaluated using the energies of the atoms and all pairs of atoms forming the system. The DIM Hamiltonian matrix is generally small and scales linearly with the number of atoms within the system. Furthermore, it requires only accurate energies of atoms and diatomic molecules which can nowadays be

obtained with advanced and reliable quantum chemistry methods. DIM methods have been succesfully employed for describing small molecules [34, 35] as well as pure and doped raregas clusters [36-39]. Here, in the spirit of the DIM, we propose to compute the ICD widths of rare-gas clusters from the widths of each pair of atoms forming the cluster.

In the section II, we give the general formulas for the computation of resonance widths from the projection-operator formalism. The standard DIM approach for the calculation of potential energy surfaces is then presented. The DIM-based method proposed here for the computation of the ICD widths is finally reported. In section III, we apply this method to the helium trimer and derive explicit DIM formulas for this system. ICD widths from the DIM approach are compared to fully ab initio (Fano-ADC) results.

\section{ICD WIDTHS IN THE PROJECTION-OPERATOR - DIM FRAMEWORK}

The general formulas for the calculation of the ICD widths are derived from the theory of resonances of Fano and Feshbach [40, 41]. The wavefunctions and coupling matrix elements involved in such calculations are then computed within the DIM approach. The main advantage of the latter is that only energies and coupling matrix elements of the atomic and diatomic fragments are needed. Computations of resonance widths in the case of electronmolecule collisions have been performed with such combined approach (so-called generalized diatomic-in-molecules approach in this context) $[43,44]$. In the present study we report a similar approach applied to the calculations of ICD widths.

\section{A. The projection-operator approach}

A general description of the Fano-Feshbach theory of resonances within the projectionoperator approach is given in [42]. We summarize here the results for a single resonance state. 
The generalization for a non-interacting manifold of resonance states is straightforward. The resonance is described by a discrete state $\left|\Psi_{d}\right\rangle$, represented by a square integrable wave function, with the energy expectation value given by

$$
E_{d}=\left\langle\Psi_{d}\left|\hat{H}_{e}\right| \Psi_{d}\right\rangle
$$

where $\hat{H}_{e}$ is the full electronic Hamiltonian. This discrete state is embedded into and coupled to a continuum of final states, which can be written as $\left|\Phi_{f k}\right\rangle=\left|\Psi_{f}\right\rangle|k\rangle$. Here, the decay channel $\left|\Psi_{f}\right\rangle$ is the eigenstate of the system after the decay with energy $E_{f}$ and $|k\rangle$ is a singleelectron continuum state. Neglecting the inter-channel coupling, the coupling between the decaying state $\left|\Psi_{d}\right\rangle$ and the final state $\left|\Phi_{f k}\right\rangle$ is

$$
V_{d, f k}=\left\langle\Psi_{d}\left|\hat{H}_{e}\right| \Phi_{f k}\right\rangle
$$

The partial ICD width for a given channel $f$ is then given by

$$
\Gamma_{f}=2 \pi\left|V_{d, f k}\right|^{2} \delta\left(E_{\mathrm{res}}-k^{2} / 2-E_{f}\right)
$$

where $E_{\text {res }}=E_{d}+\Delta\left(E_{\text {res }}\right)$ stands for the real energy of the resonance, $\Delta(E)$ is the so-called level shift. The value of $k$ is fully determined by the knowledge of $E_{d}$ and $E_{f}$. The $k$ index is thus dropped in the following. The total width is the sum of the partial ones over all decay channels

$$
\Gamma_{t o t}=\sum_{f} \Gamma_{f}
$$

\section{B. The diatomics-in-molecules approach}

The states $\left|\Psi_{d}\right\rangle,\left|\Psi_{f}\right\rangle$ and thus $V_{d, f}$ are computed within the DIM framework. In this approach, the Hamiltonian of a N-atomic system is subdivided into atomic and diatomic parts,

$$
\hat{H}_{e}=\sum_{\alpha=1}^{N-1} \sum_{\beta>\alpha}^{N} \hat{H}^{\alpha \beta}-(N-2) \sum_{\alpha=1}^{N} \hat{H}^{\alpha} .
$$

Each fragment part $\hat{H}^{\alpha}$ contains all kinetic energy operators and intra-atomic potential energy terms in $\hat{H}_{e}$ which depend only on the coordinates of the electrons assigned originally

to atom $\alpha$. The operator $\hat{H}^{\alpha \beta}$ contains all kinetic energy terms as well as intra-atomic and inter-atomic potential energy terms associated to the diatomic molecule $\alpha \beta$. 
The DIM basis set is then constructed as antisymmetrized and spin-adapted products of the atomic eigenfunctions $\left|\chi_{m}^{\alpha}\right\rangle$ with energy $e_{m}^{\alpha}$ of $\hat{H}^{\alpha}$ :

$$
\left|\Psi_{m}^{a n t i}\right\rangle=\hat{A}\left|\Psi_{m}\right\rangle=\hat{A} \hat{s} \prod_{n=1}^{N}\left|\chi_{m}^{n}\right\rangle .
$$

The operator $\hat{A}$ ensures the antisymmetrization and $\hat{s}$ creates spin-adapted linear combination of the atomic eigenfunction products. In order to evaluate the Hamiltonian matrix elements in the DIM basis set, the diatomic Hamiltonians $\hat{H}^{\alpha \beta}$ are expressed via their eigenvalues $\left\{\epsilon_{i}^{\alpha \beta}\right\}$ and eigenfunctions $\left\{\left|\psi_{i}^{\alpha \beta}\right\rangle\right\}$ :

$$
\hat{H}^{\alpha \beta}=\sum_{i}\left|\psi_{i}^{\alpha \beta}\right\rangle \epsilon_{i}^{\alpha \beta}\left\langle\psi_{i}^{\alpha \beta}\right|
$$

Noting that $\hat{A}$ and $\hat{H}^{\alpha \beta}$ commute, the operation of $\hat{H}^{\alpha \beta}$ on $\left|\Psi_{m}^{a n t i}\right\rangle$ is simply

$$
\hat{H}^{\alpha \beta}\left|\Psi_{m}^{a n t i}\right\rangle=\hat{A} \hat{H}^{\alpha \beta}\left|\Psi_{m}\right\rangle=\hat{A} \sum_{i}\left|\Psi_{i}^{\alpha \beta}\right\rangle \epsilon_{i}^{\alpha \beta}\left\langle\Psi_{i}^{\alpha \beta} \mid \Psi_{m}\right\rangle
$$

where $\left|\Psi_{i}^{\alpha \beta}\right\rangle$ is the spin-adapted product of the diatomic wavefunction $\left|\psi_{i}^{\alpha \beta}\right\rangle$ and the wavefunctions of electrons that are not included in $\hat{H}^{\alpha \beta}$,

$$
\left|\Psi_{i}^{\alpha \beta}\right\rangle=\hat{s}\left|\psi_{i}^{\alpha \beta}\right\rangle \prod_{n \neq \alpha, \beta}\left|\chi_{i}^{n}\right\rangle
$$

The overlap element $\left\langle\Psi_{i}^{\alpha \beta} \mid \Psi_{m}\right\rangle=B_{i m}^{\alpha \beta}$ can generally be determined by symmetry consideration [34, 36]. Using Eqs. (5), (6) and (8), the DIM Hamiltonian in the matrix form is

$$
\underline{\underline{H}}^{D I M}=\sum_{\alpha} \sum_{\beta} \underline{\underline{B}}^{\alpha \beta \dagger} \underline{\underline{\epsilon}}^{\alpha \beta} \underline{\underline{B}}^{\alpha \beta}-(N-2) \sum_{\alpha} \underline{\underline{e}}^{\alpha}
$$

where $\underline{\underline{\epsilon}}^{\alpha \beta}$ and $\underline{\underline{e}}^{\alpha}$ are diagonal matrices built with the diatomic and atomic fragment energies, respectively and $\underline{\underline{B}}^{\alpha \beta}$ is the overlap matrix. The electronic states of the system are then obtained by diagonalization of the DIM Hamiltonian matrix.

\section{ICD widths in the DIM approach}

We consider here a cluster constituted of $\mathrm{N}$ rare-gas $(\mathrm{Rg})$ atoms in which ICD is triggered by ionization. The system is left in one or a manifold of excited states which lie above the double ionization threshold. This is typically achieved by ionization in the inner-valence 
shell or simultaneous ionization-excitation of outer-valence electrons. This is summarized as:

$$
\operatorname{Rg}_{N} \stackrel{\text { Ionization }}{\longrightarrow}\left(\operatorname{Rg}^{+*}-\operatorname{Rg}_{N-1}\right) \stackrel{\mathrm{ICD}}{\longrightarrow}\left(\mathrm{Rg}^{+}-\mathrm{Rg}^{+}-\mathrm{Rg}_{N-2}\right)
$$

where $\mathrm{Rg}^{+*}$ illustrates that the ion is in an electronic excited state. The last term indicates that the cluster ends with two charges which may lead to two atomic ions or to larger ionic fragments.

The manifold of non-interacting resonant states $\left|\Psi_{d}\right\rangle$ and the energies are obtained in this approach by diagonalizing the DIM Hamiltonian (Eq. (10)) which is built for $R g^{+*}-R g_{N-1}$ system. We thus have

$$
\underline{\underline{E}}^{d}=\underline{\underline{U}}{\underline{\underline{H_{d}}}}^{D I M} \underline{\underline{U^{\dagger}}}
$$

where $\underline{\underline{U}}$ and $\underline{\underline{E}}^{d}$ contain the eigenvectors and eigenvalues, respectively. The matrix ${\underline{\underline{H_{d}}}}^{D I M}$ is contructed as in Eq. (10) with the energies of the $(R g-R g)$ and $\left(R g^{+*}-R g\right)$ diatomic and atomic fragments.

After the decay, the system is doubly-ionized. The diagonalization of the corresponding matrix provides the states $\left|\Psi_{f}\right\rangle(\equiv \underline{\underline{W}})$ and the associated energies $\left(\equiv \underline{\underline{E}}^{f}\right)$,

$$
\underline{\underline{E}}^{f}=\underline{\underline{W}}{\underline{\underline{H_{f}}}}^{D I M} \underline{\underline{W^{\dagger}}} .
$$

The matrix ${\underline{H_{f}}}^{D I M}$ is built using the energies of the neutral $(R g-R g)$, singly $\left(R g^{+}-R g\right)$ and doubly $\left(R g^{+}-R g^{+}\right)$ionized states of the diatomic and atomic fragments. The matrices $\underline{\underline{U}}$ and $\underline{\underline{W}}$ provide the $\left|\Psi_{d}\right\rangle$ and $\left|\Psi_{f}\right\rangle$ states within the DIM basis set, respectively. The coupling matrix between these states in the DIM approach has a similar form as Eq.(10) $[43]:$

$$
\underline{\underline{V}}_{d, f}^{D I M}=\underline{\underline{U}}\left[\sum_{\alpha} \sum_{\beta} \underline{\underline{B}}^{\alpha \beta \dagger} \underline{\underline{V}}_{d, f}^{\alpha \beta} \underline{\underline{C}}^{\alpha \beta}-(N-2) \sum_{\alpha} \underline{\underline{V}}_{d, f}^{\alpha}\right] \underline{\underline{W}}^{\dagger}
$$

where $\underline{\underline{V}}_{d, f}^{\alpha \beta}$ and $\underline{\underline{V}}_{d, f}^{\alpha}$ are constructed with the partial widths of the $\alpha \beta$ diatomic and $\alpha$ atomic fragments $\left(\Gamma_{f}\right.$, see Eqs.(2) and (3)). The matrices $\underline{\underline{B}}^{\alpha \beta}$ and $\underline{\underline{C}}^{\alpha \beta}$ are the overlap matrices (as in Eq.(10)) between the DIM basis functions (Eq.(6)) and the diatomic fragment wavefunctions (Eq.(9)) for the decaying and final states, respectively.

Within the DIM approach, it is thus only necessary to compute the couplings between the decaying and the continuum final states (Eq.(2)) of all atoms and all pairs of atoms forming the cluster. It reduces to the calculations of widths of atomic and diatomic systems which can be handled by ab initio methods already available as for instance the Fano-ADC 
approach. It should be mentioned that decay widths of large heteroatomic clusters have been estimated in a pairwise additivity approximation $[45,46]$ : the total width is given by the sum of the widths of all individual pairs forming the cluster. The DIM approach goes beyond this approximation since delocalization of the charges in the decaying and final states is taken into account. Furthermore, partial widths can also be computed within the DIM method.

\section{APPLICATION OF THE DIM APPROACH TO ICD IN HE}

\section{A. General formulas}

General formulas for the application of the DIM to compute the PES of homogeneous noble gas ionic clusters are given in [36]. In the following, we report first the DIM matrices adapted from these formulas for the states of helium trimers relevant for the ICD process: namely, ionized-excited into the $2 p$ shell $\left(\mathrm{He}^{+*}(2 p)-\mathrm{He}_{2}\right)$ and doubly-ionized $\left(\mathrm{He}^{+}(1 s)-\right.$ $\left.\mathrm{He}^{+}(1 s)-\mathrm{He}\right)$.

The DIM basis set for the ionized-excited states $\left.\left(\left\{\left.\right|^{2} \Psi_{m}^{d}\right\rangle\right\}\right)$ used here are defined as follows:

$$
\begin{aligned}
& \left|{ }^{2} \Psi_{1 x}^{d}\right\rangle=\hat{A}\left|\chi_{H e^{+}\left(2 p_{x}\right)}^{1}\right\rangle\left|\chi_{H e\left(1 s^{2}\right)}^{2}\right\rangle\left|\chi_{H e\left(1 s^{2}\right)}^{3}\right\rangle \\
& \left|{ }^{2} \Psi_{2 x}^{d}\right\rangle=\hat{A}\left|\chi_{H e\left(1 s^{2}\right)}^{1}\right\rangle\left|\chi_{H e^{+}\left(2 p_{x}\right)}^{2}\right\rangle\left|\chi_{H e\left(1 s^{2}\right)}^{3}\right\rangle \\
& \left|{ }^{2} \Psi_{3 x}^{d}\right\rangle=\hat{A}\left|\chi_{H e\left(1 s^{2}\right)}^{1}\right\rangle\left|\chi_{H e\left(1 s^{2}\right)}^{2}\right\rangle\left|\chi_{H e^{+}\left(2 p_{x}\right)}^{3}\right\rangle
\end{aligned}
$$

where $\left|\chi_{H e^{+}\left(2 p_{x}\right)}^{i}\right\rangle$ and $\left|\chi_{H e\left(1 s^{2}\right)}^{i}\right\rangle$ denote the atomic state $H e^{+}\left(2 p_{x} /{ }^{2} P_{x}\right)$ and $H e\left(1 s^{2} /{ }^{1} S\right)$ of the atom $i$ (Fig. 1), respectively. There are 6 similar DIM functions (3 for the y and $\mathrm{z}$ directions) included in the calculations. The DIM basis set for the singlet final states $\left(\left\{\left|{ }^{1} \Psi_{m}^{f}\right\rangle\right\}\right)$ are given as:

$$
\begin{aligned}
& \left.\left|{ }^{1} \Psi_{1}^{f}\right\rangle=\hat{A}\left(\left|\chi_{H e^{+}(1 s)}^{1}\right\rangle\left|\overline{\chi_{H e^{+}(1 s)}^{2}}\right\rangle\left|\chi_{H e\left(1 s^{2}\right)}^{3}\right\rangle-\left|\overline{\left.\chi_{H e^{+}(1 s)}^{1}\right\rangle}\right| \chi_{H e^{+}(1 s)}^{2}\right\rangle\left|\chi_{H e\left(1 s^{2}\right)}^{3}\right\rangle\right) / \sqrt{2}
\end{aligned}
$$

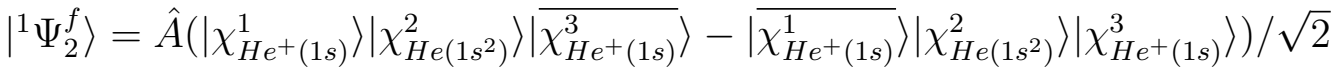

$$
\begin{aligned}
& \left|{ }^{1} \Psi_{3}^{f}\right\rangle=\hat{A}\left(\left|\chi_{H e\left(1 s^{2}\right)}^{1}\right\rangle\left|\chi_{H e^{+}(1 s)}^{2}\right\rangle\left|\overline{\left.\chi_{H e^{+}(1 s)}^{3}\right\rangle}\right\rangle-\left|\chi_{H e\left(1 s^{2}\right)}^{1}\right\rangle\left|\overline{\chi_{H e^{+}(1 s)}^{2}}\right\rangle\left|\chi_{H e^{+}(1 s)}^{3}\right\rangle\right) / \sqrt{2}
\end{aligned}
$$

where the overline indicates a spin down $(\beta)$. The expression for the triplet final states are given by the corresponding spin function.

For convenience, we introduce the following notation for the helium diatomic fragment energies used in this work: 
$S_{i j}=E_{\mathrm{He}_{2},\left(1 \Sigma_{g}^{+}\right)}\left(R_{i j}\right)$

$C_{i j}=E_{\mathrm{He}_{2}^{++},\left({ }^{1} \Sigma_{g}^{+}\right)}\left(R_{i j}\right)$

$\bar{C}_{i j}=E_{\mathrm{He}_{2}^{++},\left({ }^{3} \Sigma_{u}^{+}\right)}\left(R_{i j}\right)$

$\tilde{Q}_{i j}=\frac{1}{2}\left(E_{\mathrm{He}_{2}^{+},\left(2 \Sigma_{u}^{+}\right)}\left(R_{i j}\right)+E_{\mathrm{He}_{2}^{+},\left(2 \Sigma_{g}^{+}\right)}\left(R_{i j}\right)\right)$

$\tilde{J}_{i j}=\frac{1}{2}\left(E_{\mathrm{He}_{2}^{+},\left({ }^{2} \Sigma_{u}^{+}\right)}\left(R_{i j}\right)-E_{\mathrm{He}_{2}^{+},\left({ }^{2} \Sigma_{g}^{+}\right)}\left(R_{i j}\right)\right)$

$Q_{i j}=\frac{1}{2}\left(E_{\mathrm{He}_{2}^{+*},\left(2 \Sigma_{u}^{+}\right)}\left(R_{i j}\right)+E_{\mathrm{He}_{2}^{+*},\left({ }^{2} \Sigma_{g}^{+}\right)}\left(R_{i j}\right)\right)$

$J_{i j}=\frac{1}{2}\left(E_{\mathrm{He}_{2}^{+*},\left({ }^{2} \Sigma_{u}^{+}\right)}\left(R_{i j}\right)-E_{\mathrm{He}_{2}^{+*},\left({ }^{2} \Sigma_{g}^{+}\right)}\left(R_{i j}\right)\right)$

$\bar{Q}_{i j}=\frac{1}{2}\left(E_{\mathrm{He}_{2}^{+*},\left({ }^{2} \Pi_{g}\right)}\left(R_{i j}\right)+E_{\mathrm{He}_{2}^{+*},\left({ }^{2} \Pi_{u}\right)}\left(R_{i j}\right)\right)$

$\bar{J}_{i j}=\frac{1}{2}\left(E_{\mathrm{He}_{2}^{+*},\left({ }^{2} \Pi_{g}\right)}\left(R_{i j}\right)-E_{\mathrm{He}_{2}^{+*},\left({ }^{2} \Pi_{u}\right)}\left(R_{i j}\right)\right)$

Several analytical expressions for the ground electronic state of neutral $\mathrm{He}_{2}(S)$ have been proposed [47-49]. However, the ICD widths obtained within the DIM approach is nearly independent of the neutral potential energy curve. The diatomic fragment energies used for lowest ionized states $(\tilde{G}$ and $\tilde{U})$ are obtained from [50] and that of the ionized-excited states $(G, U, \bar{G}$ and $\bar{U})$ are taken from [51]. For the potential of the doubly-ionized dimer ( $C$ and $\bar{C}$ ) we used a purely Coulombic potential, which is correct in the interatomic distance range considered here [51].

The trimers are described in the $C_{s}$ point group and the atoms are chosen to lie in the xz-plane as shown in Fig. 1 . The vector going from atom $i$ to atom $j$ is denoted $\vec{R}_{i j}$. For the decaying states, the DIM matrices have block structure of $6 \mathrm{x} 6 \mathrm{~A}$ ' and 3x3 A" matrix :

$\underline{\underline{H_{d\left(2 A^{\prime}\right)}^{D I M}}}=\left(\begin{array}{cccccc}q_{1}+q_{2}+S_{12} & p_{1}+p_{2} & j_{2} & k_{2} & j_{1} & k_{1} \\ p_{1}+p_{2} & \bar{q}_{1}+\bar{q}_{2}+S_{12} & k_{2} & \bar{j}_{2} & k_{1} & \bar{j}_{1} \\ j_{2} & k_{2} & Q_{12}+q_{2}+S_{13} & p_{2} & J_{12} & 0 \\ k_{2} & \bar{j}_{2} & p_{2} & \bar{Q}_{12}+\bar{q}_{2}+S_{13} & 0 & \bar{J}_{12} \\ j_{1} & k_{1} & J_{12} & 0 & Q_{12}+q_{1}+S_{23} & p_{1} \\ k_{1} & \bar{j}_{1} & 0 & \bar{J}_{12} & p_{1} & \bar{Q}_{12}+\bar{q}_{1}+S_{23}\end{array}\right)$

and

$$
\underline{\underline{H_{d\left({ }^{2} A^{\prime \prime}\right)}^{D I M}}}=\left(\begin{array}{ccc}
\bar{Q}_{13}+\bar{Q}_{23}+S_{12} & \bar{J}_{23} & \bar{J}_{13} \\
\bar{J}_{23} & \bar{Q}_{12}+\bar{Q}_{23}+S_{13} & \bar{J}_{12} \\
\bar{J}_{13} & \bar{J}_{12} & \bar{Q}_{12}+\bar{Q}_{13}+S_{23}
\end{array}\right)
$$


The general form of the matrices presented above is the same as in [36]. However, the diatomic fragments energies that enter the matrix elements are that of the ionizedexcited dimer states. The singlet and triplet final states can be considered separately. The corresponding DIM matrices are 3x3 A' matrices. For the singlet states, the matrix reads:

$$
\underline{\underline{H_{f\left({ }^{1} A^{\prime}\right)}^{D I M}}}=\left(\begin{array}{ccc}
\tilde{Q}_{13}+\tilde{Q}_{23}+C_{12} & \tilde{J}_{23} & \tilde{J}_{13} \\
\tilde{J}_{23} & \tilde{Q}_{12}+\tilde{Q}_{23}+C_{13} & \tilde{J}_{12} \\
\tilde{J}_{13} & \tilde{J}_{12} & \tilde{Q}_{12}+\tilde{Q}_{13}+C_{23}
\end{array}\right)
$$

The matrix corresponding to the triplet states is obtained by replacing $C_{i j}$ by $\bar{C}_{i j}$ in the above matrix. The symbols used in the matrices are defined as follows:

$q_{i}=\bar{Q}_{i 3} \sin ^{2} \beta_{i}+Q_{i 3} \cos ^{2} \beta_{i}$

$\bar{q}_{i}=Q_{i 3} \sin ^{2} \beta_{i}+\bar{Q}_{i 3} \cos ^{2} \beta_{i}$

$p_{i}=\left(Q_{i 3}-\bar{Q}_{i 3}\right) \sin \beta_{i} \cos \beta_{i}$

$k_{i}=\left(J_{i 3}-\bar{J}_{i 3}\right) \sin \beta_{i} \cos \beta_{i}$

$j_{i}=\bar{J}_{i 3} \sin ^{2} \beta_{i}+J_{i 3} \cos ^{2} \beta_{i}$

$\bar{j}_{i}=J_{i 3} \sin ^{2} \beta_{i}+\bar{J}_{i 3} \cos ^{2} \beta_{i}$

where $\beta_{i}$ defines the angle between $\vec{R}_{i 3}$ and $\vec{R}_{12}$ (see Fig. 1 ).

The atomic energies are not included in the above matrices. They lead to a global energy shift and do not play a role in the calculations of the widths: if the energy of the neutral cluster is set to zero, the energy shift of the decaying states is $\mathrm{E}\left(\mathrm{He}^{+}(\mathrm{n}=2)\right)=65.4 \mathrm{eV}$ and that of the final states is $2 \mathrm{E}\left(\mathrm{He}^{+}(1 s)\right)=49.1 \mathrm{eV}$.

The diatomic coupling matrix elements are taken from [51] and are used as follows:

$\Lambda_{i j}^{S+}=\frac{1}{2 \sqrt{\pi}}\left(\sqrt{\Gamma^{2 \Sigma_{g}^{+} \rightarrow^{1} \Sigma_{g}^{+}}\left(R_{i j}\right)}+\sqrt{\left.\Gamma^{2 \Sigma_{u}^{+} \rightarrow^{1} \Sigma_{g}^{+}\left(R_{i j}\right)}\right)}\right.$

$\bar{\Lambda}_{i j}^{S+}=\frac{1}{2 \sqrt{\pi}}\left(\sqrt{\Gamma^{2} \Pi_{g} \rightarrow^{1} \Sigma_{g}^{+}\left(R_{i j}\right)}+\sqrt{\Gamma^{2} \Pi_{u} \rightarrow^{1} \Sigma_{g}^{+}\left(R_{i j}\right)}\right)$

$\Lambda_{i j}^{S-}=\frac{1}{2 \sqrt{\pi}}\left(-\sqrt{\Gamma^{2} \Sigma_{g}^{+} \rightarrow^{1} \Sigma_{g}^{+}\left(R_{i j}\right)}+\sqrt{\Gamma^{2 \Sigma_{u}^{+} \rightarrow^{1} \Sigma_{g}^{+}}\left(R_{i j}\right)}\right)$

$\bar{\Lambda}_{i j}^{S-}=\frac{1}{2 \sqrt{\pi}}\left(\sqrt{\Gamma^{2} \Pi_{g} \rightarrow^{1} \Sigma_{g}^{+}\left(R_{i j}\right)}-\sqrt{\Gamma^{2} \Pi_{u} \rightarrow^{1} \Sigma_{g}^{+}\left(R_{i j}\right)}\right)$

where the superscript $\mathrm{S}$ denotes singlet final states. Similar matrix elements are defined for the triplet final states.

It should be noted that intra-atomic decay channels are energetically closed in helium clusters. The atomic coupling matrix elements are thus set to zero. The DIM coupling matrices for the singlet final states are : 


$$
\underline{\underline{V}}_{2}^{D I M} A^{\prime}{ }_{1}^{1} A^{\prime}=\underline{\underline{U_{2} A^{\prime}}}\left(\begin{array}{ccc}
0 & \Lambda_{13}^{S+} \cos \beta_{1}-\bar{\Lambda}_{13}^{S+} \sin \beta_{1} & \Lambda_{23}^{S-} \cos \beta_{2}-\bar{\Lambda}_{23}^{S-} \sin \beta_{2} \\
0 & \Lambda_{13}^{S+} \sin \beta_{1}+\bar{\Lambda}_{13}^{S+} \cos \beta_{1} & \Lambda_{23}^{S-} \sin \beta_{2}+\bar{\Lambda}_{23}^{S-} \cos \beta_{2} \\
\Lambda_{12}^{S-} & 0 & \Lambda_{23}^{S+} \cos \beta_{2}-\bar{\Lambda}_{23}^{S+} \sin \beta_{2} \\
\bar{\Lambda}_{12}^{S-} & 0 & \Lambda_{23}^{S+} \sin \beta_{2}+\bar{\Lambda}_{23}^{S+} \cos \beta_{2} \\
\Lambda_{12}^{S+} & \Lambda_{13}^{S-} \cos \beta_{1}-\bar{\Lambda}_{13}^{S-} \sin \beta_{1} & 0 \\
\bar{\Lambda}_{12}^{S+} & \Lambda_{13}^{S-} \sin \beta_{1}+\bar{\Lambda}_{13}^{S-} \cos \beta_{1} & 0
\end{array}\right){\underline{W_{1} A^{\prime}}}^{\dagger}
$$

and

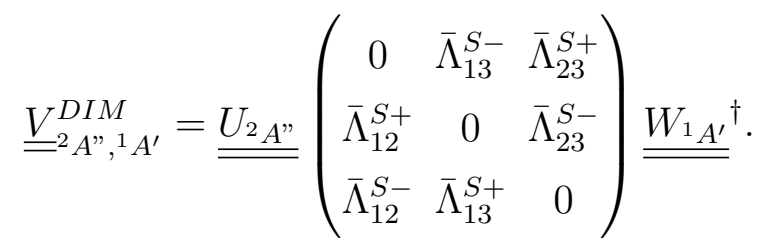

The DIM coupling matrices for the triplet final states are obtained by replacing in the above matrices the diatomic fragment coupling matrix elements by that corresponding to the triplet.

\section{B. Total decay widths}

The three helium atoms in the trimer are weakly bound such that the average interatomic distance is about $10.4 \AA[52,53]$ and there is no preferential equilibrium geometry [53, 54]. In this study, we have investigated the trimer in equilateral and isosceles geometry. As shown in Fig. 1, the three atoms lie on the xz plane and the distance between the two atoms 1 and 2 is kept fixed at $R_{12}=4 \AA$. The distance between the center of mass of the two fixed atoms and the third atom is denoted Q.

The electronic states of the trimer are labeled according to their spatial symmetry classification in the $C_{s}$ point group (A' or A"), their spin multiplicity (doublet for the decaying states and singlet/triplet for the final states) but also according to the symmetry of the asymptotic atomic and diatomic fragments. For example, ${ }^{2} \mathrm{~A}^{\prime}\left({ }^{1} \Sigma_{g}^{+} /{ }^{2} P_{x}\right)$ is used for the decaying state that converges at large $\mathrm{Q}$ to $\mathrm{He}_{2}\left({ }^{1} \Sigma_{g}^{+}\right)$and $\mathrm{He}^{+*}\left({ }^{2} P_{x}\right)$. The label ${ }^{2} \mathrm{~A}^{\prime}\left({ }^{2} \Pi_{u} /{ }^{1} S\right)$ is employed for the decaying state corresponding to $\mathrm{He}_{2}^{+*}\left({ }^{2} \Pi_{u}\right)-\mathrm{He}\left(1 \mathrm{~s}^{2} /{ }^{1} S\right)$. The final state converging to singlet $\mathrm{He}_{2}^{+}\left({ }^{2} \Sigma_{g}^{+}\right)$and $\mathrm{He}^{+}\left({ }^{2} S\right)$ is labeled ${ }^{1} \mathrm{~A}^{\prime}\left({ }^{2} \Sigma_{g}^{+} /{ }^{2} S\right)$ and that converging to $\mathrm{He}_{2}^{2+}\left({ }^{1} \Sigma_{g}^{+}\right)-\mathrm{He}\left(1 \mathrm{~s}^{2} /{ }^{1} S\right)$ is named ${ }^{1} \mathrm{~A}^{\prime}\left({ }^{1} \Sigma_{g}^{+} /{ }^{1} S\right)$, for instance. 
The total decay widths for each decaying electronic states at an equilateral geometry $\left(\mathrm{R}_{12}=\mathrm{R}_{13}=\mathrm{R}_{23}=4 \AA\right)$ are shown in table I. Details on the Fano-ADC calculations and a thorough discussion of decay widths in helium trimer as well as a comparison with helium dimer data can be found in [55]. The widths obtained from the DIM calculations differ from the $a b$ initio Fano-ADC results by at most 20\%. Owing to the complexity to evaluate accurate resonance widths, such agreement is satisfactory.

The dependence of the total decay widths with respect to $\mathrm{Q}$ (see Fig. 1) is shown and compared to the Fano-ADC results in Figs. 2 and 3. Results for the A' states (Fig. 2) of the DIM approach compare quantitatively with that of the Fano-ADC calculations for $\mathrm{Q}$ above 2-3 $\AA$ (i.e. $\mathrm{R}=2.8-3.6 \AA$ ). For smaller distances, the agreement is less satisfactory. This is is due to the limited DIM basis set used and the stronger 3-body effects, expected at short interatomic distances, not accounted for by the DIM approach. However, the DIM results agree well in the relevant interatomic distance range since the mean He-He distance in clusters goes from $10.4 \AA$ (trimer) to $3.6 \AA$ (superfluid liquid helium) [39]. The DIM calculations reproduces well the widths for the A" states (Fig. 3), even at short interatomic distances. The corresponding orbitals are perpendicular to the trimer plane and therefore overlap less than that for the A' states, which explains the better agreement.

\section{Partial decay widths and limit of the DIM approach}

We have shown that the DIM approach can be succesfully employed to compute accurate total decay widths. In order to give a full description of ICD, the partial widths to each of the singlet and triplet final states must be well reproduced within the DIM approach. The DIM partial widths to these states for two A" decaying states are compared to the Fano-ADC results in Figs. 4 and 5.

The partial widths for the A" $\left({ }^{1} \Sigma_{g}^{+} /{ }^{2} \mathrm{P}_{y}\right)$ decaying state are well reproduced by the DIM approach. The partial widths corresponding to the deexcitation of the $\operatorname{He}^{+}\left({ }^{2} \mathrm{P}_{y}\right)$ and ionization of $\mathrm{He}_{2}$ show a $1 / \mathrm{R}^{6}$ behavior as expected by the virtual photon exchange mechanism $[56,57]$. The width corresponding to the double ionization of the $\mathrm{He}_{2}$ is at least 2 order of magnitude smaller. This channel corresponds to Electron-Transfer-Mediated-Decay (ETMD) $[58,59]$ in which one electron from $\mathrm{He}_{2}$ is transferred to $\mathrm{He}^{+}$and a second electron from $\mathrm{He}_{2}$ is ionized. This process is efficient only at short interatomic distances for which 
significant spatial orbital overlap is possible.

For the A" $\left({ }^{2} \Pi_{g} /{ }^{1} S\right)$ decaying state, the DIM approach reproduces well the asymptotic widths for the dominant channel (i.e. two-site double ionization of the $\mathrm{He}_{2}$ ) but fails for the weakest ones (i.e. ionization of the third helium atom). For the ${ }^{2} A^{\prime \prime}\left({ }^{2} \Pi_{g} /{ }^{1} S\right) \rightarrow$ ${ }^{1} A^{\prime}\left({ }^{2} \Sigma_{u}^{+} /{ }^{2} S\right)$ transition, the DIM underestimates the asymptotic widths by a factor of 2 . Furthermore, the ab initio widths for the ${ }^{2} A^{\prime \prime}\left({ }^{2} \Pi_{g} /{ }^{1} S\right) \rightarrow{ }^{1} A^{\prime}\left({ }^{2} \Sigma_{g}^{+} /{ }^{2} S\right)$ transition exhibit a $1 / \mathrm{R}^{10}$ behavior demonstrating a dipole-forbidden transition. Indeed, as shown in [55], the decay to these channels is asymptotically mediated by a quadrupole-quadrupole interaction. In this limit, the deexcitation of the dimer from ${ }^{2} \Pi_{g}$ to ${ }^{2} \Sigma_{g}^{+}$is dipole-forbidden. This is a 3-body effect that is not accounted for in the DIM approach and represents a limit to the calculations of partial ICD widths with the DIM method. It should, however, be noted that these channels are always weak and do not significantly contribute to the decay. Same conclusions are drawn for the partial widths of the last A" state and that of the $A^{\prime}$ states (not shown). The DIM approach can therefore be used to describe ICD in rare-gas clusters.

\section{CONCLUSION}

A method combining the projection-operator approach of resonant scattering theory and the diatomics-in-molecules technique is reported. This combined approach is applied to the helium trimer for which working formula are explicited. The total ICD widths and the dominant partial widths obtained from this approach compare quantitatively to the full $a b$ initio Fano-ADC results over a large set of geometries. Disagreements between some weak channels are explained by strong three-body effects which are not accounted for in the DIM method. The present approach has fairly low computational costs since the DIM matrices for the decaying and final states are small even for systems having hundreds of atoms. It therefore constitutes an efficient tool for studying polyatomic clusters and paves the way to a complete description of ICD in large systems.

\section{ACKNOWLEDGEMENTS}

This work has been financially supported by the Czech Science foundation (Project GČR P208/12/0521). N.S. acknowledges financial state aid managed by the Agence Nationale 
de la Recherche, as part of the programme Investissements d'avenir under the reference ANR-11-IDEX-0004-02.

[1] Non-Hermitian Quantum Mechanics, N. Moiseyev, Cambridge University Press (2011).

[2] G. Gamow, Z. Phys. 51, 204 (1928).

[3] L. S. Cederbaum, J. Zobeley and F. Tarantelli, Phys. Rev. Lett. 79, 4778 (1997).

[4] S. Marburger, O. Kugeler, U. Hergenhahn and T. Möller, Phys. Rev. Lett. 90, 203401 (2003).

[5] T. Jahnke et al. Phys. Rev. Lett. 93, 163401 (2004).

[6] M. Mucke et al. Nature Physics 6, 143 (2010).

[7] T. Jahnke et al. Nature Physics 6, 139 (2010).

[8] V. Averbukh et al. Dynamical Processes in Atomic and Molecular Physics 29, Bentham Science Publishers (2012).

[9] V. Averbukh et al. J. Electron Spectrosc. Relat. Phenom. 183, 36 (2011).

[10] U. Hergenhahn, J. Electron Spectrosc. Relat. Phenom. 184, 78 (2011).

[11] T. Jahnke, J. Phys. B: Atomic, Molecular and Optical Physics 48, 082001 (2015).

[12] T. Pflüger, A. Senftleben, X. Ren, A. Dorn and J. Ullrich, Phys. Rev. Lett. 107, 223201 (2011).

[13] H.K. Kim et al. PNAS 108, 11821 (2011).

[14] H.K. Kim et al. Phys. Rev. A 88, 042707 (2013).

[15] H.K. Kim et al. Phys. Rev. A 89, 022704 (2014).

[16] T. Jahnke et al. Phys. Rev. Lett. 99, 153401 (2007).

[17] N. Sisourat et al. Nature Physics 6, 508 (2010).

[18] V. Averbukh and P. Kolorenč, Phys. Rev. Lett. 103, 183001 (2009).

[19] A.I. Kuleff, K. Gokhberg, S. Kopelke and L.S. Cederbaum, Phys. Rev. Lett. 105, 043004 (2010).

[20] S.D. Stoychev, A.I. Kuleff, F. Tarantelli and L.S. Cederbaum, J. Chem. Phys. 129, 074307 (2008).

[21] P.V. Demekhin, S. Scheit, S.D. Stoychev and L.S. Cederbaum, Phys. Rev. A 78, 043421 (2008).

[22] K. Kreidi et al. Phys. Rev. A 78, 043422 (2008). 
[23] T. Ouchi et al. Phys. Rev. A 83, 053415 (2011).

[24] K. Gokhberg, P. Kolorenč, A.I. Kuleff and L.S. Cederbaum, Nature 505, 661 (2014).

[25] F. Trinter et al. Nature 505, 664 (2014).

[26] R. Santra and L.S. Cederbaum, Phys. Rep. 368, 1 (2002).

[27] N. Vaval and L. S. Cederbaum, J. Chem. Phys. 126, 164110 (2007).

[28] A. Ghosh, S. Pal and N. Vaval, J. Chem. Phys. 139, 064112 (2013).

[29] A. Ghosh, S. Pal and N. Vaval, Mol. Phys. 112, 669 (2014).

[30] V. Averbukh and L.S. Cederbaum, J. Chem. Phys. 123, 204107 (2005).

[31] V. Averbukh and L.S. Cederbaum, J. Chem. Phys. 125, 094107 (2006).

[32] E. Fasshauer, P. Kolorenč and M. Pernpointner J. Chem. Phys. 142, 144106 (2015).

[33] F.O. Ellison, J. Am. Chem. Soc. 85, 3540 (1963).

[34] J.C. Tully, J. Chem. Phys 58, 1396 (1973).

[35] J.C. Tully, J. Chem. Phys 59, 5122 (1973).

[36] P.J. Kuntz and J. Valldorf, Z. Phys. D Atoms, Molecules and Clusters 8, 195 (1988).

[37] R.B. Gerber, D. Shemesh, M.E. Varner, J. Kalinowski and B. Hirshberg, Phys. Chem. Chem. Phys. 16, 9760 (2014) (and references therein).

[38] D. Bonhommeau, A. Viel and N. Halberstadt, J. Chem. Phys. 120, 11359 (2004).

[39] D. Bonhommeau, M. Lewerenz and N. Halberstadt, J. Chem. Phys. 128, 054302 (2008).

[40] U. Fano, Phys. Rev. 124, 1866 (1961).

[41] H. Feschbach, Rev. Mod. Phys. 36, 1076 (1964).

[42] W. Domcke, Phys. Rep. 208, 97 (1991).

[43] A.K. Belyaev, A.S. Tiukanov and W. Domcke, Phys. Rev. A 65, 012508 (2001).

[44] A.K. Belyaev, A.S. Tiukanov and W. Domcke, Phys. Scr. 80, 048124 (2009).

[45] N.V. Kryzhevoi, V. Averbukh and L.S. Cederbaum, Phys. Rev. B 76, 094513 (2007).

[46] E. Fasshauer, M. Förstel, S. Pallmann, M. Pernpointner and U. Hergenhahn, New J. Phys. 16, 103026 (2014).

[47] R.A. Aziz and M.J. Slaman, J. Chem. Phys. 94, 8047 (1991).

[48] K. T. Tang, J. P. Toennies and C. L. Yiu, Phys. Rev. Lett. 74, 1546 (1995).

[49] M. Przybytek, W. Cencek, J. Komasa, G. Łach, B. Jeziorski and K. Szalewicz, Phys. Rev. Lett. 104, 183003 (2010).

[50] J. Xie, B. Poirier and G. I. Gellene, J. Chem. Phys. 122, 184310 (2005). 
[51] P. Kolorenč, N. V. Kryzhevoi, N. Sisourat and L. S. Cederbaum, Phys. Rev. A 82, 013422 (2010).

[52] M. Lewerenz, J. Chem. Phys. 106, 4596 (1997).

[53] J. Voigtsberger, S. Zeller, J. Becht, N. Neumann, F. Sturm, H.-K. Kim, M. Waitz, F. Trinter, M. Kunitski, A. Kalinin, J. Wu, W. Schöllkopf, D. Bressanini, A. Czasch, J.B. Williams, K. Ullmann-Pfleger, L. Ph H. Schmidt, M.S. Schöffler, R.E. Grisenti, T. Jahnke and R. Dörner, Nat. Comm. 5, 5765 (2014).

[54] D. Bressanini and G. Morosi, J. Phys. Chem. A 115, 10880 (2011).

[55] P. Kolorenč and N. Sisourat, J. Chem. Phys. 143, 224310 (2015).

[56] V. Averbukh, I.B. Müller and L.S. Cederbaum, Phys. Rev. Lett. 93, 263002 (2011).

[57] T. Jahnke et al. Phys. Rev. Lett. 99, 153401 (2007).

[58] J. Zobeley, R. Santra and L.S. Cederbaum, J. Chem. Phys. 115, 5076 (2001).

[59] K. Sakai et al. Phys. Rev. Lett. 106, 033401 (2011). 
Figure 1. Geometry of the trimer : the atoms lie on the xz plane. The distance between the atoms $\mathrm{A}$ and $\mathrm{B}$ is kept fixed at $\mathrm{R}_{12}=4 \AA$.

\begin{tabular}{|c|c|c|}
\hline & $\Gamma_{D I M}\left(10^{-5}\right.$ a.u. $)$ & $\Gamma_{\text {Fano-ADC }}\left(10^{-5}\right.$ a.u. $)$ \\
\hline${ }^{2} \mathrm{~A}^{\prime}\left({ }^{1} \Sigma_{g}^{+} /{ }^{2} \mathrm{P}_{x}\right)$ & 1.21 & 1.46 \\
\hline${ }^{2} \mathrm{~A}^{\prime}\left({ }^{1} \Sigma_{g}^{+} /{ }^{2} \mathrm{P}_{z}\right)$ & 3.09 & 2.70 \\
\hline${ }^{2} \mathrm{~A}^{\prime}\left({ }^{2} \Sigma_{g}^{+} /{ }^{1} S\right)$ & 2.89 & 2.67 \\
\hline${ }^{2} \mathrm{~A}^{\prime}\left({ }^{2} \Sigma_{u}^{+} /{ }^{1} S\right)$ & 2.89 & 2.74 \\
\hline${ }^{2} \mathrm{~A}^{\prime}\left({ }^{2} \Pi_{g} /{ }^{1} S\right)$ & 1.29 & 1.49 \\
\hline${ }^{2} \mathrm{~A}^{\prime}\left({ }^{2} \Pi_{u} /{ }^{1} S\right)$ & 1.29 & 1.46 \\
\hline \hline${ }^{2} \mathrm{~A}^{\prime \prime}\left({ }^{1} \Sigma_{g}^{+} /{ }^{2} \mathrm{P}_{y}\right)$ & 0.83 & 0.85 \\
\hline${ }^{2} \mathrm{~A}^{\prime \prime}\left({ }^{2} \Pi_{g} /{ }^{1} S\right)$ & 0.80 & 0.83 \\
\hline${ }^{2} \mathrm{~A}^{\prime \prime}\left({ }^{2} \Pi_{u} /{ }^{1} S\right)$ & 0.90 & 0.89 \\
\hline
\end{tabular}

Table I. Total decay widths at equilateral geometry $\left(\mathrm{R}_{12}=\mathrm{R}_{13}=\mathrm{R}_{23}=4 \AA\right)$ obtained with the DIM and Fano-ADC approaches. 


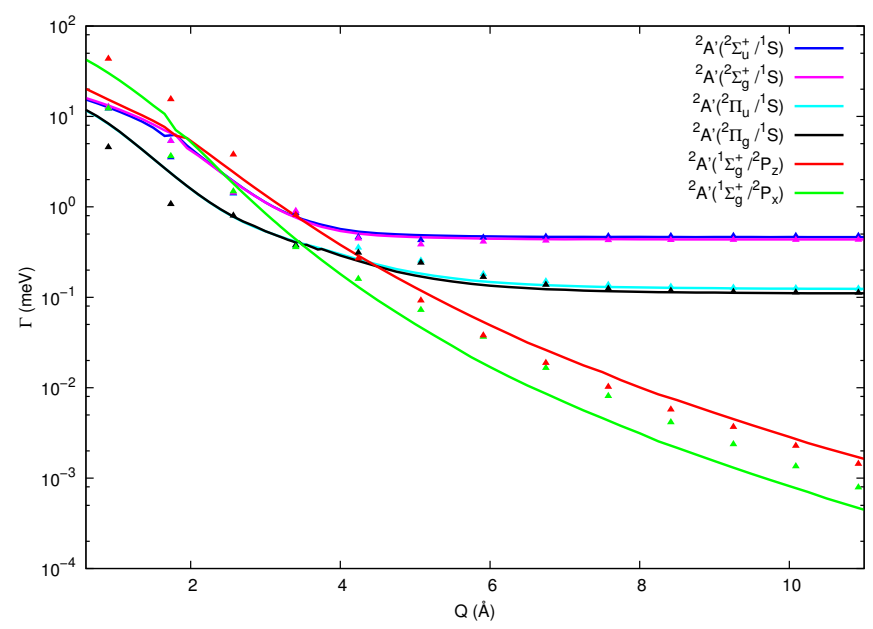

Figure 2. Total widths for the A' decaying states. DIM and Fano-ADC results are shown in dots and in lines, respectively.

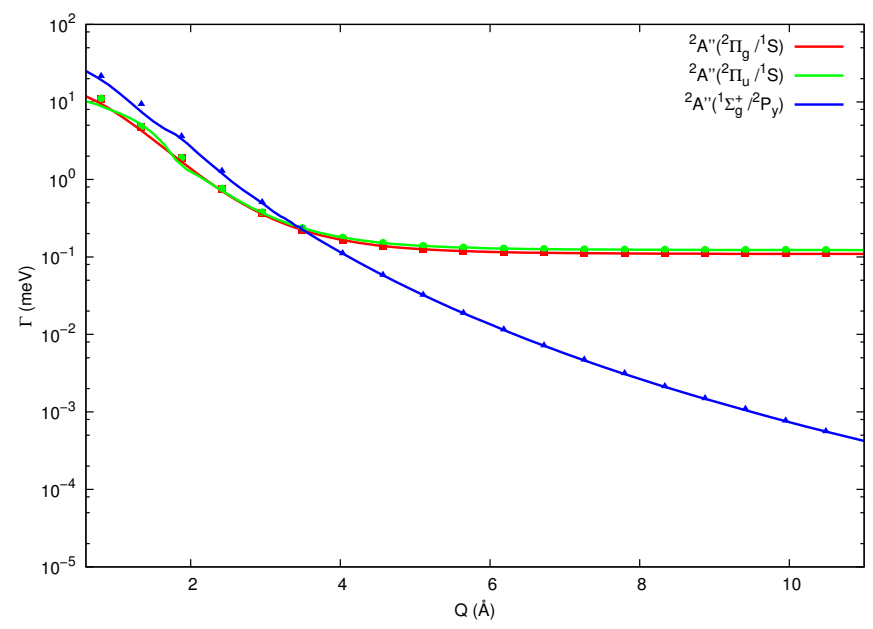

Figure 3. Total widths for the A" decaying states. DIM and Fano-ADC results are shown in dots and in lines, respectively. 


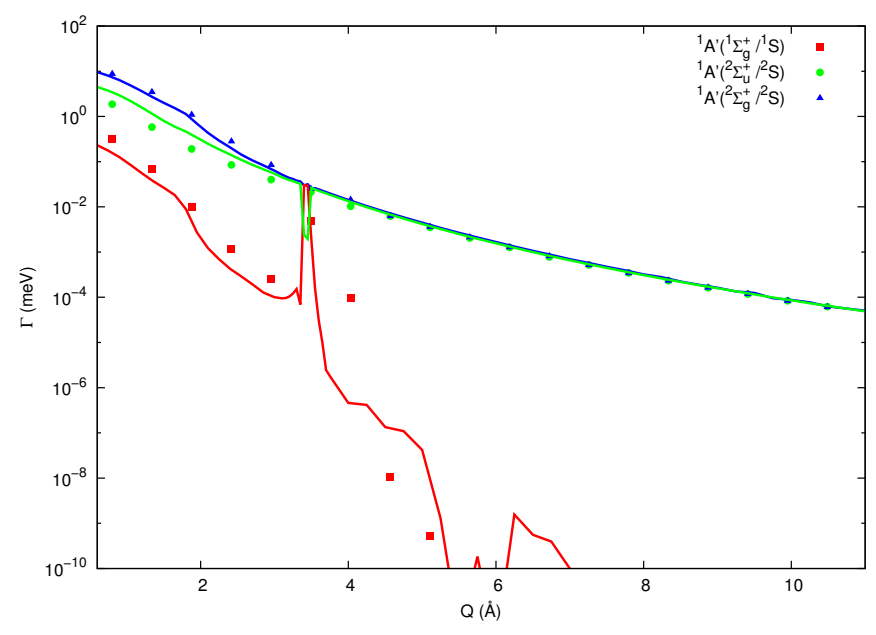

Figure 4. Partial widths to the singlet final states for the $\mathrm{A}^{\prime \prime}\left({ }^{1} \Sigma_{g}^{+} /{ }^{2} \mathrm{P}_{y}\right)$ decaying state. DIM and Fano-ADC results are shown in dots and in lines, respectively. The sharp peaks around $\mathrm{Q}=3.5 \AA$ are attributed to strong mixing between the decay channels near equilateral geometry [55].

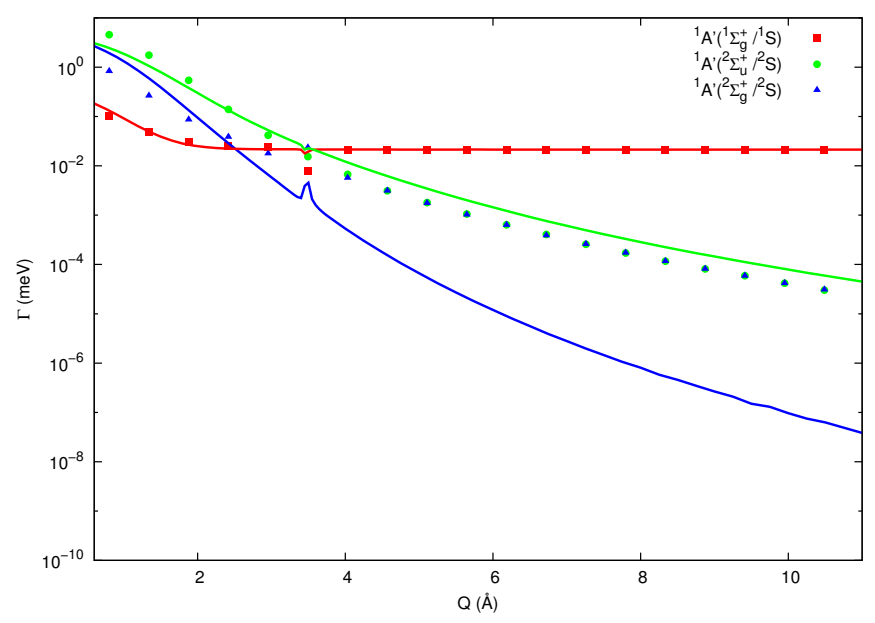

Figure 5. Partial widths to the singlet final states for the $\mathrm{A} "\left({ }^{2} \Pi_{g} /{ }^{1} S\right)$ decaying state. DIM and Fano-ADC results are shown in dots and in lines, respectively. The sharp peaks around $\mathrm{Q}=3.5 \AA$ are attributed to strong mixing between the decay channels near equilateral geometry [55]. 